\title{
Using a 3-D multicellular simulation of spinal cord injury with live cell imaging to study the neural immune barrier to nanoparticle uptake
}

\author{
Alan P. Weightman ${ }^{\S}$, Stuart I. Jenkins ${ }^{\S}$, and Divya M. Chari ( $\left.\square\right)$ \\ Cellular and Neural Engineering Group, Institute for Science and Medicine, Huxley Building, Keele University, Keele, ST5 5BG, UK \\ $\S$ These authors contributed equally to this work.
}

\begin{abstract}
Development of nanoparticle (NP) based therapies to promote regeneration in sites of central nervous system (CNS; i.e. brain and spinal cord) pathology relies critically on the availability of experimental models that offer biologically valid predictions of NP fate in vivo. However, there is a major lack of biological models that mimic the pathological complexity of target neural sites in vivo, particularly the responses of resident neural immune cells to NPs. Here, we have utilised a previously developed in vitro model of traumatic spinal cord injury (based on 3-D organotypic slice arrays) with dynamic time lapse imaging to reveal in real-time the acute cellular fate of NPs within injury foci. We demonstrate the utility of our model in revealing the well documented phenomenon of avid NP sequestration by the intrinsic immune cells of the CNS (the microglia). Such immune sequestration is a known translational barrier to the use of NP-based therapeutics for neurological injury. Accordingly, we suggest that the utility of our model in mimicking microglial sequestration behaviours offers a valuable investigative tool to evaluate strategies to overcome this cellular response within a simple and biologically relevant experimental system, whilst reducing the use of live animal neurological injury models for such studies.
\end{abstract}

\section{Introduction}

Nanotherapeutic strategies for neurological injury and disease (in areas such as neuroimaging, drug and gene delivery) are gaining increasing popularity in the burgeoning field of nanomedicine, particularly applications involving nanoparticles (NPs) [1-4].
Extensive efforts have been focused on developing strategies to deliver NP-based therapeutic agents into sites of neurological pathology. Most studies focusing on development of nanoparticle based therapeutics for neurological injury/disease usually address delivery of such agents across the protective blood brain barrier surrounding neural tissue. However the majority of 
such studies have neglected to consider the role of the immune component of the nervous system itself. From research in recent years it has become apparent that such delivery strategies will need to take account of a major barrier to therapeutic NP delivery imposed by these resident immune cells of the brain and spinal cord-namely the "microglia" [5-7]. Microglia are highly phagocytic and motile immune cells present throughout the central nervous system (CNS), and are involved in constant surveillance of their local microenvironment for identification and removal of "foreign" material $[7,8]$. Microglia have been shown to dominate uptake versus other neural cell types due to their comparatively rapid and extensive particle uptake profiles [9]. This has been demonstrated for different NP types, using defined glial co-cultures [9], undefined co-cultures of primary neurons and glial cells, and more complex transverse organotypic spinal cord slices (intact tissue) co-cultured with peripheral nerve grafts [10]. Together, it is estimated that the glial cells account for up to $99.5 \%$ of cellular uptake of NPs, with microglia shown to be responsible for the majority of this uptake in vivo [11]. The high clinical relevance of this phenomenon is reflected in in vivo studies that replicate these observations of microglial dominance of uptake following direct delivery of NPs to the CNS [11-13]. Injection of aminosilanecoated superparamagnetic iron oxide NPs (core diameter $15 \mathrm{~nm}$ ) into glioblastoma tumours for thermotherapy reveals that the NPs are overwhelmingly localised in microglia/macrophagelike cells [13]. In striking con- trast, other cell types in the brains of these patients, such as the target glioblastoma cells and reactive astro- cytes rarely displayed intracellular NPs. This result is not due to tumour cells lacking the capacity for NP uptake, as experiments using isolated gliosarcoma and glioma cells in vitro (i.e. in the absence of microglial cells) demonstrate that tumour cells can take up NPsobservations which would have erroneously predicted efficient tumour cell uptake in the intact CNS [12]. Similarly, when injected into sites of transecting spinal cord injury (SCl) in rodents, Spherofluor NPs (approx. $360 \mathrm{~nm}$ diameter) showed localisation exclusively to immune cells (our unpublished data). We have also shown that in line with their phagocytic roles, microglia inactivate particles by sequestration into vesicles and extensive lysosomal degradation [14].

Such observations highlight an important clinical challenge confronting NP delivery into the CNS particularly for sites of neurological injury and disease where large numbers of activated microglia exist. They additionally highlight the need for the development of multicellular experimental model systems, wherein post-injury pathological processes and the microglial barrier can be effectively replicated, for the developmental testing of therapeutic approaches to overcome the barrier imposed by microglial clearance. Live animal neurological injury models represent the most biologically relevant experimental systems in this context, but the relative inaccessibility of the brain and spinal cord for experimental manipulation necessitate highly invasive procedures with several attendant ethical and practical drawbacks [11, 15-17]. There is therefore a major need for alternative, biomimetic models to Reduce, Refine and Replace live animal use in neuro-nanotechnology studies [18]. Despite this need, studies demonstrating competitive microglial clearance and the related barrier to uptake in other neural cell types have generally used simple, undefined co-culture systems [10] which overall fail to replicate the complex cytoarchitecture of neural tissue [19]. It should also be noted that all of these previous studies did not incorporate a neurological injury component and are therefore limited in their ability to mimic the intricate environment of neurological injury sites, wherein multiple cell types with specialised functions co-exist.

In this context, a novel in vitro model of SCl described by ourselves recently could provide a technically facile but valuable experimental tool for studying microglial particle uptake dynamics within an injury-simulated environment [20]. This model is based on the use of 3-D spinal cord tissue arrays within which a focal, traumatic injury can be induced severing the nerve fibres, mimicking for example injuries due to knife/gunshot wounds. We previously demonstrated that major pathological features of neurological injury in vivo, such as the formation of a glial cell scar and an age related decline in nerve fibre regeneration were all mimicked in the injury foci- highlighting the model's relevance to CNS injury in vivo [20]. Importantly, injury foci were found to 
be progressively infiltrated by microglial cells which showed highly activated phenotypes characteristic of pathological sites. Despite the neuro-mimicry offered by the model, it is not known whether it can be used to replicate competitive sequestration of NPs by microglia in neural pathology sites, or to mimic intercellular differences in NP uptake profiles as seen in vivo. If such a demonstration can be shown to be successful, then our model could provide a powerful tool to study the microglial barrier and investigate pharmaceutical approaches to overcome it. Here, we have delivered a well-characterised NP into spinal cord slice injury sites; using a combination of dynamic live-cell and confocal imaging, we document cellular particle uptake and sequestration profiles by the microglia versus other major neural cell populations in the simulated injury foci.

\section{Materials and methods}

The care and use of animals was in accordance with the Animals (Scientific Procedures) Act of 1986 (UK) with local ethics committee approval.

\subsection{Reagents and equipment}

Tissue culture plastics, media and supplements were from Fisher Scientific (Loughborough, UK) and Sigma Aldrich (Poole, UK). Millicell culture inserts (PICM0RG50) and Omnipore membranes (JHWP04700) were from Millipore (Watford, UK). Spherofluor (Sphero ${ }^{\mathrm{TM}}$ ) magnetic NPs (comprising a polystyrene core stained with the fluorescent dye Nile red, within a composite layer of polystyrene and iron oxide, coated with carboxylic acid groups) were obtained from Spherotech Inc. (Lake Forest, Illinois, USA). The NP used in this study has been shown to accumulate in areas of transecting $\mathrm{SCl}$ (the in vivo counterpart to the injury model used in the current study and which is associated with breakdown of the blood-spinal cord barrier) after intravenous delivery in rodents [21], and has been well-characterised by electron microscopy, zetasizer analysis, powder X-ray diffraction and Fourier transform infrared spectroscopy $(200-390 \mathrm{~nm}$ diameter, mean $360 \mathrm{~nm}$; $-14 \mathrm{mV}$ zeta-potential; iron content $15 \%-20 \% \mathrm{w} / \mathrm{v}$ ) [14]. The live/dead cell viability kit was from Invitrogen (Paisley, UK). Vectashield mounting medium containing 4',6-diamidino-2phenylindole (DAPI) was from Vector Laboratories (Peterborough, UK). Primary antibodies were: rat anti-CD11b (clone 5C6, MCA711GT; Bio-Rad, UK), rabbit anti-C $\mathrm{X}_{3} \mathrm{Cr} 1$ (TP501; amsbio, UK), rabbit anti-glial fibrillary acidic protein (GFAP) from DakoCytomation (Ely, UK), rat anti-myelin basic protein (MBP) from Serotech (Kidlington, UK) and rabbit anti-neuronal class III $\beta$-tubulin (clone TUJ-1) from Covance (Princeton, NJ). Fluorescein isothiocyanate (FITC)conjugated secondary antibodies were from Jackson ImmunoResearch Laboratories Inc. (West Grove, PA, USA), except FITC-conjugated antibiotin secondary antibody, which along with biotinconjugated lectin (from Lycopersicon esculentum) and dexamethasone (DEX) 21-phosphate disodium (D4902; $\geq 97 \%$ pure) was from Sigma Aldrich (Poole, UK).

\subsection{Preparation and lesioning of organotypic spinal cord slice cultures}

Spinal cord slice cultures were generated and lesioned as described previously $[20,22,23]$. Briefly, mouse pups aged 1-3 postnatal days (P1-3) were terminally anaesthetised, decapitated and a midline incision was made along the length of each spine. The spinal cords (approximately $10 \mathrm{~mm}$ in length) were rapidly dissected out from the thoracolumbar region and placed in ice-cold slicing medium (Earle's balanced salt solution, EBSS, buffered with $25 \mathrm{mM}$ HEPES). The cords were transferred onto the chopping plate of a Mcllwain tissue chopper and sliced lengthways in the parasagittal plane $(350 \mu \mathrm{m}$ thickness) to produce five to seven usable slices per cord. Intact slices were transferred to Omnipore membranes, resting on the Millicell culture insert membrane within $35 \mathrm{~mm}$ Petri dishes (Fig. 1(a)(1)). Slices were cultured at the air- medium interface with an $80 \%$ medium change every two days $(50 \%$ modified Eagles' medium (MEM), 25\% heatinactivated horse serum, 25\% EBSS supplemented with $36 \mathrm{mM}$ D-glucose, $100 \mathrm{U} \cdot \mathrm{mL}^{-1}$ penicillin, $100 \mathrm{\mu g} \cdot \mathrm{mL}^{-1}$ streptomycin and $250 \mathrm{ng} \cdot \mathrm{mL}^{-1}$ amphotericin B). Cultures were incubated in 5\% $\mathrm{CO}_{2} / 95 \%$ humidified air at $37{ }^{\circ} \mathrm{C}$ for a total of six days in vitro (DIV). 
(a) (1)

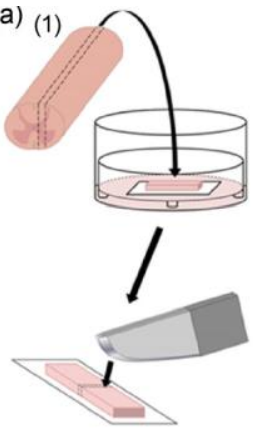

(2)

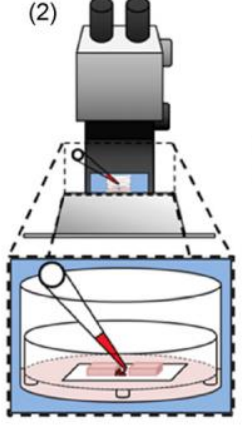

(3)

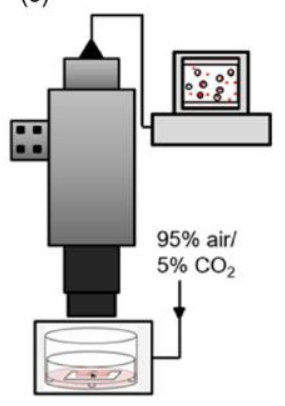

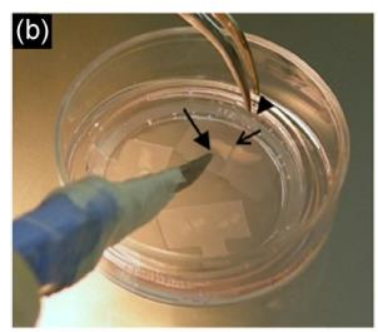

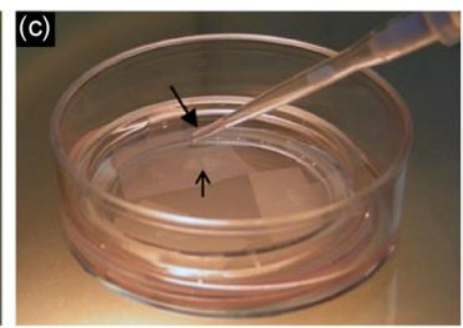

(d)

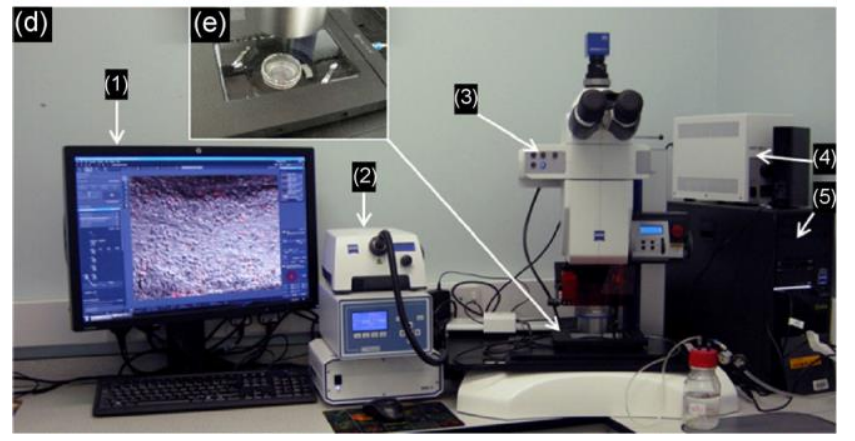

Figure 1 Experimental setup for the time-lapse recording of NP within lesions of spinal cord slice cultures. (a) Schematic diagram depicting the experimental manipulations performed in the study.

(1) Mouse spinal cords were sliced in the longitudinal parasagittal plane and cultured within inserts for a total of 6 DIV. Slices were lesioned at 1 DIV using a double-bladed scalpel. (2) NPs were added into slice lesions at 6 DIV using a P2 Gilson pipette and a dissection microscope at $\times 12.5$ magnification. (3) Slices were maintained in an incubation chamber, and imaged for 6-24 h using an upright time-lapse microscope. (b) Photograph demonstrating the procedure of lesioning slices (open arrow) with a double- bladed scalpel (arrow), within slice culture inserts. A "tab" of culture membrane (arrowhead) was clamped against the insert wall using forceps to keep the slice stationary. (c) Photograph showing the addition of NPs in a pipette tip (arrow) to the lesions of slices (open arrow) within culture inserts. (d) The time-lapse recording setup consisting of: (1) computer monitor showing a recording in progress; (2) controllers for (from top downwards) the brightfield lightsource, incubator thermoregulator, and microscope stage/column; (3) upright time-lapse microscope with motorised stage/column and incubator with regulated temperature and gas supply; (4)

UV lightsource; and (5) computer. (e) The incubation chamber in situ, containing slices. 
The lesioning procedure (Figs. 1(a)(1) and 1(b)) was conducted within a laminar flow hood using a dissec- tion microscope at $\times 12.5$ magnification. Slices were focally lesioned using a double-bladed scalpel (size 15 surgical blades) with small lateral movements made to ensure the complete severance of nerve fibre tracts. The slice debris between the two lesion margins was subsequently removed using an aspirator, fitted with a $200 \mu \mathrm{L}$ pipette tip, leaving well-defined lesion margins (verified by microscopic analysis) approximately $440 \mu \mathrm{m}$ apart. This allows for easy visualisation of cellular NP uptake dynamics within the injury site. Spinal cord slices were lesioned at 1 DIV then main- tained until 6 DIV for all experiments, as a peak in microglial infiltration has previously been shown by ourselves to occur at five days postlesioning [20].

\subsection{Delivery of NPs into lesioned slices}

A dissection microscope at $\times 12.5$ magnification and a manual pipette were used to deliver Sphero NPs (suspended in fresh slice culture medium at $20 \mu \mathrm{g} \cdot \mathrm{mL}^{-1} ; 0.75 \mu \mathrm{L}$ dose) into the lesion of each slice (Figs. 1(a)(2) and 1(c)). The feasibility of using an automated NP delivery device (i.e. Nanoject IITM Auto- Nanoliter Injector) was assessed (data not shown), but due to the prolonged setup time and additional experimental manipulations required, a manual pipette was considered sufficient for this study. Slices were incubated with NPs for $6 \mathrm{~h}$, as substantial cellular uptake occurs within this time period [14, 24, 25]. All slices were fixed in $4 \%$ paraformaldehyde (PFA; 20 min; room temperature (RT)). The NP concentration and volume applied was optimised in pilot experiments, as volumes greater than $1 \mu \mathrm{L}$ resulted in lateral spread of NPs around the lesioned slice, and NP concentrations lower than $20 \mu \mathrm{g} \cdot \mathrm{mL}^{-1}$ limited the NPs available for cellular uptake within the lesion site, hindering quantification of uptake (data not shown).

\subsection{Time-lapse imaging experiments}

Lesioned spinal cord slices (within $35 \mathrm{~mm}$ Petri dishes) at six DIV were placed in an incubation chamber (preheated to $37{ }^{\circ} \mathrm{C} ; 5 \% \quad \mathrm{CO}_{2} / 95 \%$ humidified air; Figs. 1(a)(3), 1(d) and 1(e)) on an upright time-lapse microscope (Axiozoom V16, AxioCam ICm1 camera; 
Carl Zeiss, Germany). Time-lapse imaging commenced within 10 min of NP addition. Lesioned slices without NPs were similarly recorded at 6 DIV to observe the cellular activity within injury sites. For all experiments, transmitted light images (auto exposure; average $6 \mathrm{~ms}$ ) were captured every $2 \mathrm{~min}$, with counterpart fluorescence imaging (fixed at $50 \mathrm{~ms}$ ) for NP addition experiments. Z-stack imaging was used at each time- point to allow compensation for the limited focal drift observed in pilot experiments. Video files from each imaging session were created using ZEN software (Blue Ed., v.1.1.1.0, Carl Zeiss, Germany) and Premiere Elements 7 (Adobe, USA).

\subsection{Assessment of lesioned slice viability post- live cell imaging}

To assess whether the imaging procedures had adverse effects on slice viability, control lesioned slices without NPs were stained following time-lapse imaging experi- ments $(n=4)$ and DEX treatment $(n=3)$. The high viability (ca. 96\%) of control unlesioned slices was previously demonstrated. Slices were washed three times in phosphate buffered saline (PBS) and incubated for $15 \mathrm{~min}$ at $37^{\circ} \mathrm{C}$ with calcein $\left(1 \mu \mathrm{L} \cdot \mathrm{mL}^{-1}\right)$ and ethi- dium homodimer $\left(3 \mu \mathrm{L} \cdot \mathrm{mL}^{-1}\right)$ to label live and dead cells respectively. Slices were washed three times in PBS and mounted in Vectashield mounting medium with DAPI. Consistent camera exposure settings were used for both fluorescent channels, with images converted to grayscale. Corrected integrated density measurements were performed using ImageJ software (version 1.45s; $\mathrm{NIH}$ ) and the values for the live stained micrograph expressed as a percentage of the sum total from both [20, 26].

\subsection{DEX treatment of slices to induce microglial suppression}

For microglial suppression experiments, an $80 \%$ medium change was performed at 1 DIV and every two days thereafter with culture medium containing DEX $(10 \mu \mathrm{M}$ final concentration). This concentration is within the range that has previously been shown to maximally affect various cell types in vitro [27, 28]. Additionally, $5 \mu \mathrm{L}$ of DEX culture medium was applied daily directly into slice lesions to ensure sustained delivery of drug within the lesion site. This small volume was used to limit the quantity of medium covering the slices, a critical factor in the maintenance of slice viability at the air-medium interface $[22,23]$. The overall viability of live/dead stained control slices treated with DEX was quantified to assess the safety of the method of treatment.

\subsection{Comparative NP incubations with isolated high purity astrocyte populations}

NP uptake experiments have previously been performed in our lab using high purity astrocyte cultures (ca. 98\% GFAP+) derived from cerebral cortices of neonatal Sprague-Dawley rats using an established procedure [29]. Briefly, enzymaticallydetached astro- cytes were plated on poly-D-lysine (PDL)-coated cover- slips $\left(4 \times 10^{4}\right.$ cells $\left.\cdot \mathrm{cm}^{-2}\right)$ in D10 medium (Dulbecco's modified Eagle's medium, 1 $\mathrm{mM}$ sodium pyruvate, $50 \mathrm{mg} \cdot \mathrm{mL}^{-1}$ streptomycin, 50 $\mathrm{U} \cdot \mathrm{mL}^{-1}$ penicillin, $10 \%$ fetal bovine serum and $2 \mathrm{mM}$ glutaMAX-I) at $37{ }^{\circ} \mathrm{C}$ in humidified $5 \% \mathrm{CO}_{2} / 95 \%$ air. After $24 \mathrm{~h}$, Sphero NPs were added at $20 \mathrm{ng} \cdot \mathrm{mL}^{-1}$ and incubated for $4 \mathrm{~h}$, then fixed and immunocytochemically stained as detailed below. The date from these experiments was used to demonstrate the differences in NP uptake when using isolated purified cells, versus those in the injury simulated environment containing microglia.

\subsection{Immunocytochemistry}

Slices were washed three times in PBS before and after fixation with 4\% PFA (20 min; RT). Samples were incubated in blocker consisting of $5 \%$ normal donkey serum (10\% for lectin staining) in PBS (with $0.3 \%$ Triton $\mathrm{X}-100$ for TUJ-1, MBP and GFAP staining), with lectin $(1: 200$ in blocker) or primary antibodies (TUJ-1 1:1,000; MBP 1:200; GFAP 1:500; CD11b 1:125; $\mathrm{Cx}_{3} \mathrm{Cr} 1$

$1: 100 ; 24 \mathrm{~h}$ at $\left.4{ }^{\circ} \mathrm{C}\right)$. Following three PBS washes, samples were incubated with appropriate FITCconjugated secondary antibodies ( $4 \mathrm{~h}$ at RT or 4 $\left.{ }^{\circ} \mathrm{C}\right)$. Slices were subsequently washed three times with PBS and mounted with Vectashield mounting medium containing DAPI.

\subsection{Fluorescence imaging: Fixed slices}

Slices were visualised at $\times 200$ magnification on an 
Axio Scope A1 fluorescence microscope (Carl Zeiss Microlmaging GmbH; Germany) fitted with an Axio Cam ICc1 digital camera and AxioVision software. Where applicable, fluorescence images of immunostained slices were merged/stitched using Photoshop CS5.1 (version 12.1) allowing assessment of entire lesions.

\subsection{Quantification of microglial infiltration ( \pm DEX treatment)}

The total number of lectin-reactive (lectin+) microglia within each lesion was divided by the total area of the lesion $\left(\mathrm{mm}^{2}\right)$ to account for differences in slice width (dorsal-ventral). Average values for microglial density were calculated for lesions within DEX-treated and control slices ( $n \geq 4$ per group).

\subsection{Assessment of extent of NP uptake within lesioned slices within microglia and astrocytes}

NP-labelled astrocytes and microglia (GFAP+ and lectin-reactive respectively; the neural cell types with the most extensive particle uptake profiles in isolated cell cultures) were counted within lesion areas and expressed as a percentage of the total number of cells ( $n \geq 3$ per group). The extent of NP accumulation in individual cells was assessed semiquantitatively by comparing the area of NP accumulation with the aver- age cross-sectional area of an astrocyte or microglial nucleus, as appropriate using a previously validated method for nanoparticle uptake quantification [14]. The extent of NP uptake by each cell was scored as: "low" (less than $10 \%$ of the average area of a nucleus); "medium" (10\%-50\%); or "high" (>50\%). Only NPs that were clearly intracellular were included in analyses.

\subsection{Statistical analysis}

The number of experiments, $n$, refers to slices obtained across different animals and litters. Prism v5.0 software (GraphPad, USA) was used for statistical analyses. Data are expressed as mean \pm standard error of the mean (SEM). Live/dead viability analysis: Student's $t$-test (two-tailed) with Welch's correction was used to compare viability of lesioned slices following recording or DEX treatment. Microglial infiltration analysis: Student's t-test (twotailed) was used to compare microglial infiltration \pm DEX treatment. NP uptake analysis: Data were square root transformed and a oneway analysis of variance (ANOVA) conducted using Bonferroni's multiple comparison test for post-hoc analysis (a Bartlett's test was performed to examine homogeneity of variance) to detect differences in astrocyte and microglial NP uptake \pm DEX treatment. Extent of NP uptake analysis: Student's $t$-test (twotailed) with Welch's correction was used to detect differences in the extent of astrocyte and microglial NP uptake \pm DEX treatment.

\section{Results}

\subsection{Validation of NP delivery and time-lapse recording methodology}

The use of a pipette for NP delivery into spinal cord slices proved to be a simple and effective method to achieve focal particle delivery into lesion sites (Fig. 2(a)). A dissection microscope facilitated accurate guidance of the pipette tip to the lesion site, and live assessment of the distribution of NP solution with respect to the lesion site. Fluorescence micrographs of live/dead stained slices captured immediately following time-lapse experiments (Fig. 2(b)) revealed a high proportion of viable cells in the body of the slice with the few dead cells typically located around the slice edges and in the vicinity of the lesion site, suggesting the procedure is without acute adverse effects. Indeed, lesioned slice viability following time- lapse recording experiments was $95.8 \% \pm 2.0 \%$ (live/ dead assay; $n=4$ ), which is in accordance with our previous values for slices maintained in conventional incubators [20].

\subsection{Time-lapse recordings of NP uptake by cells within slice lesions and identification of cell phenotype}

Recordings of control slices 5 days post-lesion induction revealed a population of highly motile cells with a rounded morphology within the margins of lesions (Video S1 in the Electronic Supplementary Material (ESM)) suggestive of a microglial phenotype; these cells also appeared to be highly proliferative (Fig. 3(a) and Video S2 in the ESM) with frequent cell divisions 

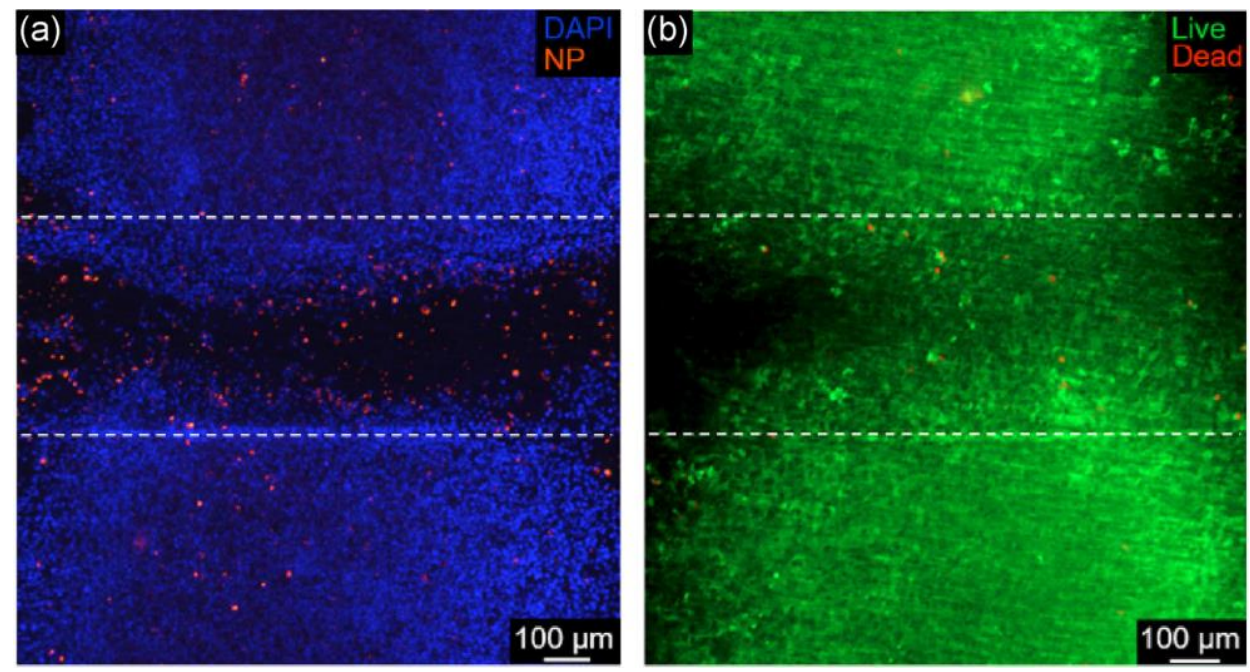

Figure 2 Validation of NP delivery and slice viability for time-lapse recording experiments. Representative fluorescence micrographs of lesioned slices, generated at P1, lesioned at 1 DIV and fixed at 6 DIV (white broken lines demarcate margins). (a) Nuclear staining (DAPI) reveals lesion margins, and demonstrates focal delivery of NPs into the lesion site. (b) Live/dead-staining shows high viability in a lesioned slice following $6 \mathrm{~h}$ time-lapse recording.
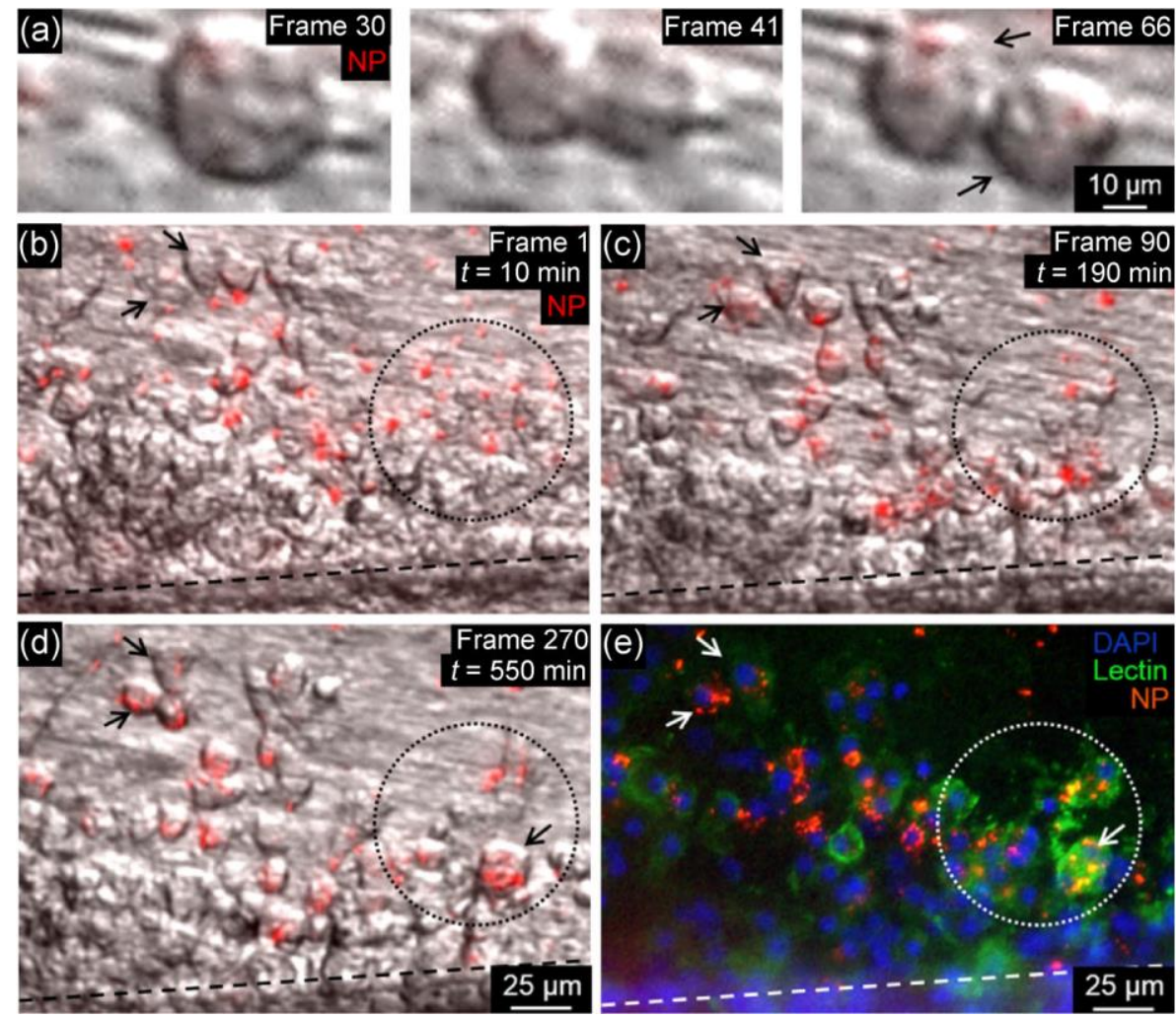

Figure 3 Introduction of NPs into spinal cord lesions results in rapid and extensive NP accumulation by microglia. (a) Merged transmitted light and fluorescence micrographs of dividing microglia within the lesion margins of a P1-derived slice (lesioned at 1 DIV, fluorescent NPs added 5 days later and incubated for $6 \mathrm{~h}$ ), with individual frame numbers corresponding to Video S2 in the ESM. (b)-(d) Merged transmitted light and fluorescence micrographs (different slice to (a); 10, 190 and 550 min post-particle addition), with individual frame numbers corresponding to Video S3 in the ESM. Cells displaying morphologies and activity typical of microglia predominate within the lesion and demonstrate a time-dependent increase in particle accumulation (black arrows and dotted circle). (e) A fluorescent micrograph of the same field as (b)-(d) (arrows indicate same cells), fixed at $600 \mathrm{~min}$ post-particle addition, shows that the marked cells are lectin-reactive microglia. Note the rounded morphology of lectin-reactive cells displaying large NP accumulations. 
observed. Following the addition of NPs into lesion sites, rounded highly active cells could be observed to respond to particle addition with highly motile activity and engulfment with evidence of rapid membrane reorganisation (extrusion and withdrawal). This resulted in high accumulation of NPs by this cell population, and the extent of intracellular particle accumulations could clearly be seen to increase over the duration of the recordings (Figs. 3(b)-3(d) and Video S3 in the ESM). Post-imaging, it proved simple to identify cells whose behaviours were recorded in imaging studies through immunocytochemical staining for cell type-specific markers. This was achieved by marking (prior to fixation) the lesion edge from which recordings were made and noting distinctive anato- mical features at the margin, including curvature of lesions, distinctive NP clusters and so on. Staining of slices post-recording clearly revealed this rounded motile population with prominent engulfing behaviours to be lectin-reactive microglia (Fig. 3(e) and Video S3 in the ESM).

\subsection{Immunocytochemical staining of lesioned slices to identify cell populations showing NP internalisation}

Detailed analyses were conducted in order to identify specific cell sub-types associated with NP uptake within lesions. In keeping with findings from time-lapse recordings (Fig. 3(e)), lectin-reactive microglia in slice lesions displayed large accumulations of NPs and had rounded morphologies, indicative of microglial activation (Fig. 4(a)). In contrast the microglia found within the body of slices displayed multiple ramified processes characteristic of "resting" (non-activated) cells (Fig. 4(b)). Microglial identity was confirmed
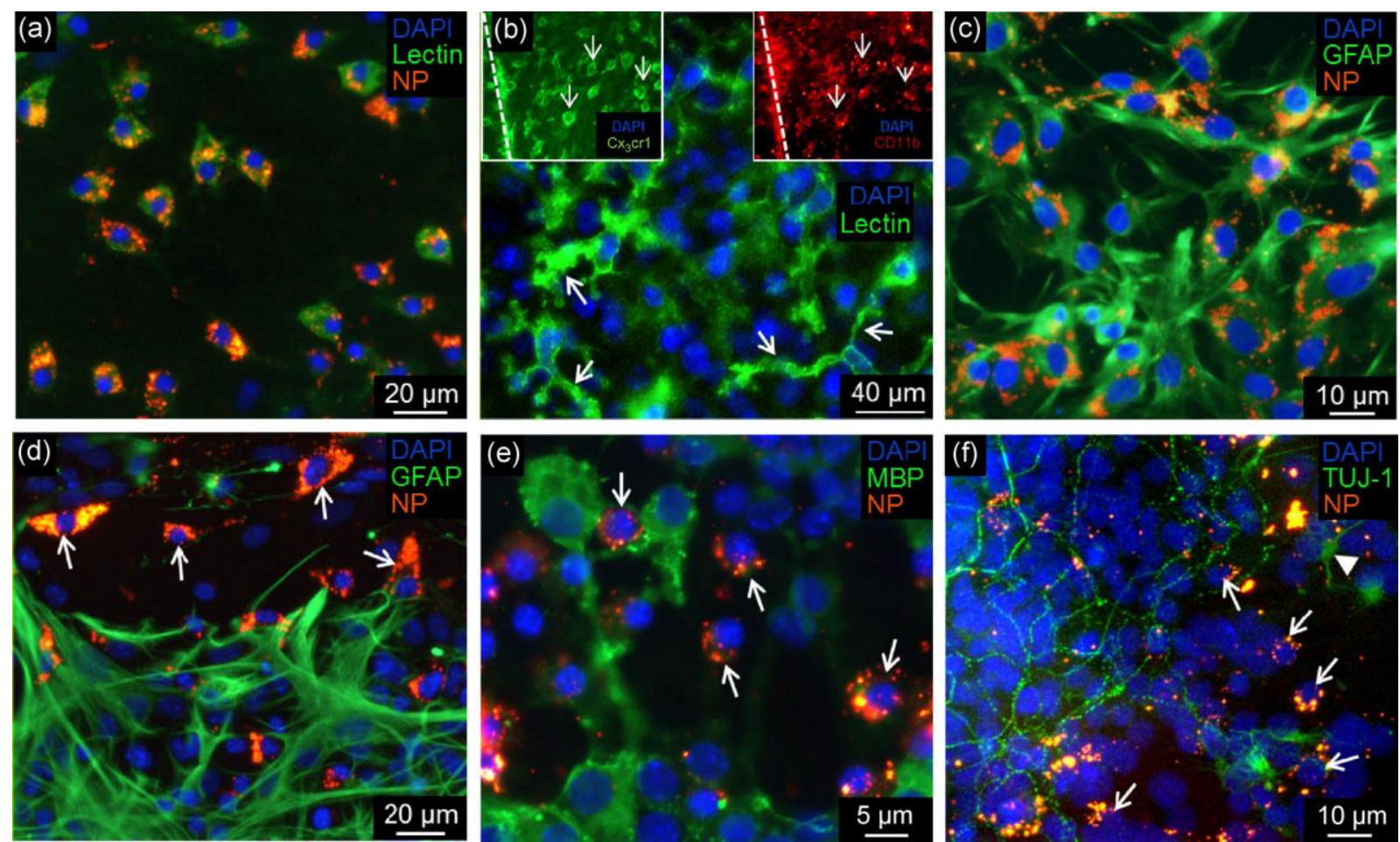

Figure 4 Microglia outcompete other neural cells for NPs in the multicellular environment of slice lesion sites. (a) Microglia in lesion sites take up NPs avidly and display characteristically large accumulations. Note the rounded morphologies associated with phenotypically "activated" microglia. (b) By contrast, microglia within the body of the slice exhibited ramified morphologies (arrows), typical of an "unactivated" state. For comparison, insets show rounded, amoeboid cells within a lesion (line marks lesion edge), immunostained with

microglial markers $\mathrm{Cx}_{3} \mathrm{Cr} 1$ and $\mathrm{CD} 11 \mathrm{~b}$ (arrows indicate same cells in each image). (c) Astrocytes in high purity isolated cultures (propagated on tissue culture plastic) exhibit large accumulations of Sphero NPs $\left(20 \mu \mathrm{g} \cdot \mathrm{mL}^{-1}, 4 \mathrm{~h}\right)[18,22]$. (d) GFAP ${ }^{+}$cells show little evidence of NP uptake with NP localisation in rounded GFAP ${ }^{-}$cells. (e) No evidence of NP uptake was observed in $\mathrm{MBP}^{+}$oligodendrocytes. (f) Very limited evidence of NP uptake was observed in the cell bodies of TUJ-1+ neurons (e.g. arrowhead). For (d)-(f), note that the surrounding $\mathrm{GFAP}^{-} / \mathrm{MBP}^{-} / \mathrm{TUJ}-1^{-}$cells exhibit large NP accumulation, characteristic of microglia (arrows). 
using three specific markers: lectin, $\mathrm{Cx}_{3} \mathrm{Cr} 1$ and $\mathrm{CD} 11 \mathrm{~b}$ (Fig. 4(b)). Astrocytes in high purity 2-D cultures have previously been shown by us to extensively internalise Sphero NPs, following comparable incubation periods [14, 25] (same NP concentration; also see Fig. 4(c) for internalisation by astrocytes in isolated, high purity primary-derived cultures). By contrast, astrocytes in slice cultures exhibited far lower NP uptake levels (in terms of both proportions of cells labelled and the extent of NP accumulation). Indeed, overall there was little evidence of NP uptake by the GFAP+ astrocytes within the multicellular environment of the slice lesions, including at the lesion margins (Fig. 4(d)). Similarly, MBP+ oligodendrocytes (Fig. 4(e)) and TUJ$1^{+}$neurons (Fig. 4(f)), showed negligible particle uptake; in every instance, NP accumulations were overwhelmingly associated with cells that were negative for markers of these major neural cell types, highlighting that delivered NPs were almost exclusively localised to the microglial population.

\subsection{Dexamethasone treatment reduces the infiltration and NP uptake profiles of microglia}

DEX treatment of lesioned slices did not affect overall viability (live/dead assay; $>97 \%$ fluorescence intensity being attributable to calcein (live cells) in all slices; two-tailed Student's $t$ test versus lesioned slices without DEX ; $n=3$ ), with the number of dead cells within and around the lesion site being similar to controls (Fig. 5(a)). Immunocytochemical staining of DEX treated lesioned slices showed low numbers of lectin-reactive microglia in lesion sites (Fig. 5(b)).
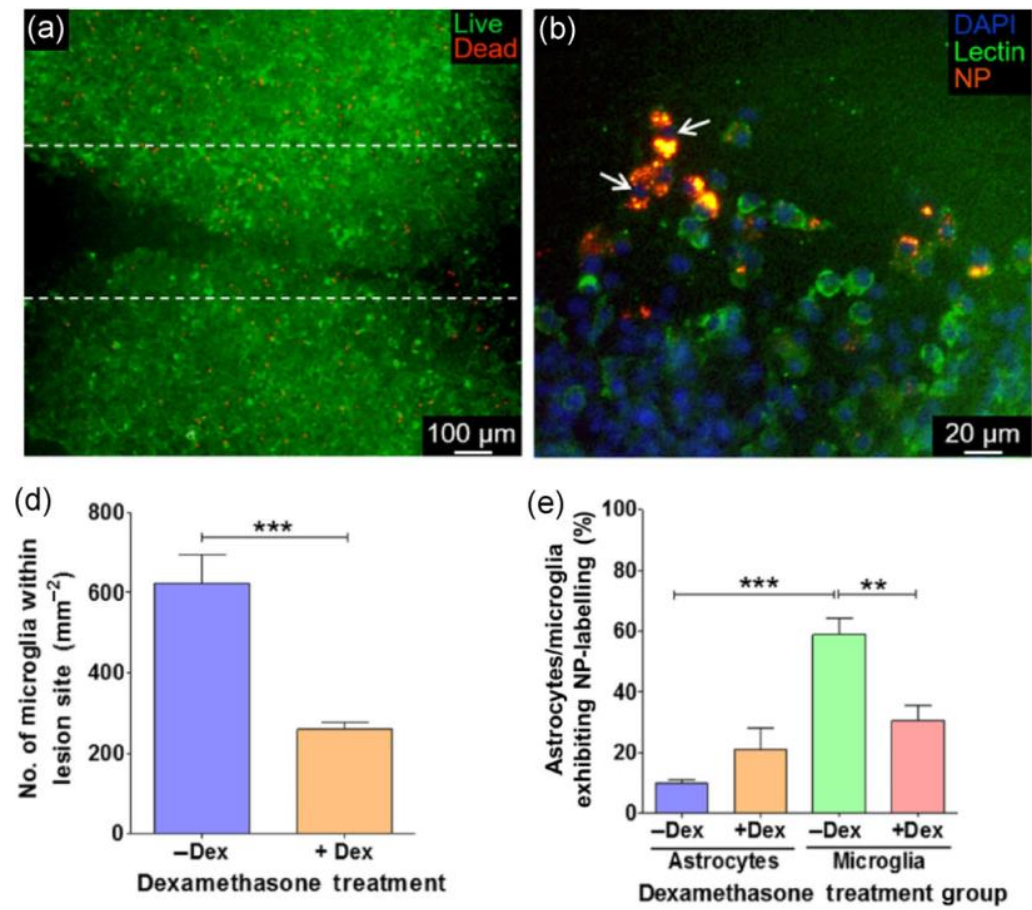
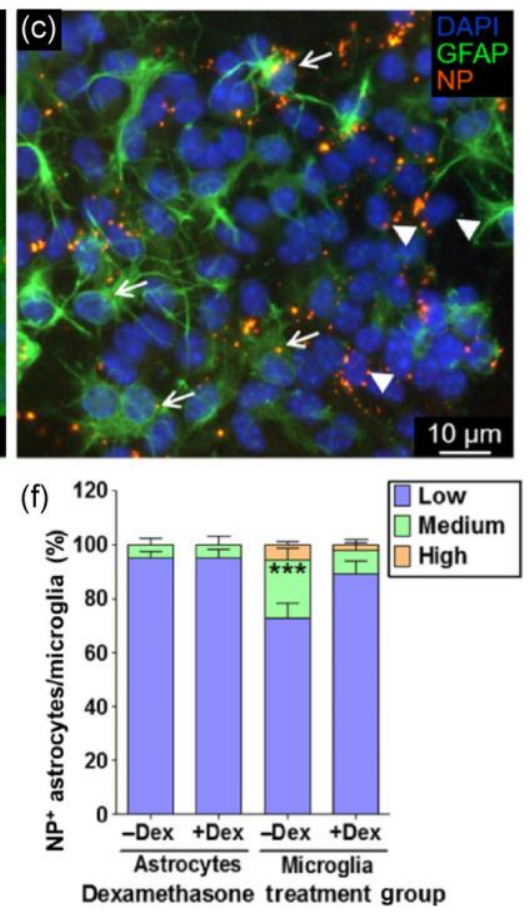

Figure 5 Semi-quantitative evaluation of the effect of DEX treatment on microglial uptake of NPs compared to astrocytes. (a) Representative fluorescence micrograph of a live/dead-stained lesioned slice following 5 days of DEX treatment. (b) Representative fluorescence micrograph showing reductions in both the number of lectin-reactive microglia and extent of NP accumulation in lesions. Occasionally, microglia with large accumulations were apparent (arrows). (c) Representative fluorescence micrograph of DEX-treated slice showing $\mathrm{GFAP}^{+}$astrocytes with a low level of NP labelling (arrows). GFAP ${ }^{-}$ cells (arrowheads, pointing leftwards) typically exhibited lower levels of NP uptake than GFAP' cells in untreated controls. (d) Bar graph showing a significant reduction in the number of lectin-reactive microglia within the lesions following 5 days of DEX treatment $\left.{ }^{* * *} p<0.001 ; n \geq 4\right)$. (e) Bar graph showing that, in lesions, a significantly greater proportion of microglia than astrocytes exhibited NP uptake ${ }^{* * *} p<0.001 ; n \geq 3$ per group). A smaller percentage of microglia were NP-labelled in DEX-treated slices $\left({ }^{* *} p<0.01\right)$. (f) Bar graph comparing the extent of NP uptake by microglia and astrocytes in DEXtreated slices and controls. The majority of labelled astrocytes exhibited low levels of NP accumulation (regardless of DEX treatment) with no astrocytes showing high levels of accumulation. Microglia exhibited low, medium and high levels of NP accumulation, with the proportion of medium labelling being significantly reduced by DEX treatment ${ }^{* \star *} p<0.001 ; n \geq 3$ per group). 
Occasionally, microglial cells were observed with high levels of NP accumulation, comparable to slices without DEX (arrows in Fig. 5(b)), but fewer cells exhibited NP uptake overall (compare Fig. 5(b) with Fig. 4(a)). Compared to control slices without DEX, there was greater evidence of NP uptake by GFAP+ astrocytes in lesions (compare Fig. 5(c) with Fig. 4(d)). Quantification of the extent of microglial infiltration into lesions showed reduced numbers of microglia following DEX treatment compared to untreated controls (Fig. 5(d)). A quantitative analysis of the proportions of microglia and astrocytes displaying NP labelling within lesion sites in the absence of DEX treatment (Fig. 5(e)) showed that a significantly greater percentage of microglia was labelled than astrocytes (ca. 5-fold more). In DEX treated slices, the proportion of microglia exhibiting NP uptake was significantly reduced. This percentage was still greater than the proportion of astrocytes labelled on average. Further, while visual observations revealed that astrocytes showed larger accumulations in DEX treated cultures, this was not found to be statistically significant. A semi-quantitative analysis of the extent of particle accumulation in NP-labelled cells (Fig. 5(f)) shows predominantly "low" levels in astrocytes, with $<5 \%$ of cells showing "medium" and no astrocytes exhibiting "high" levels of accumulation: This pattern of labelling did not change with DEX treatment. In comparison to astrocytes, microglia displayed cells with "high" and "medium" levels of labelling, with the percentage of cells exhibiting "medium" levels being significantly reduced with DEX treatment.

\section{Discussion}

Sequestration of particles delivered to the CNS parenchyma by the resident neural immune cells (the microglia), represents a major translational challenge confronting the delivery of NP-based therapeutics to areas of neurological injury and disease. As microglial sequestration is a critical determinant of the eventual fate of administered particles, experimental research critically requires model systems that simulate microglial responses to delivered therapeutic particles within a pathological environment. Here, we demonstrate the utility of our novel $\mathrm{SCl}$ model to examine the interactions of major neural cell types (that play key roles in injury and repair) with NPs within a site of neurological injury. Notably, we were able to demonstrate for the first time in real-time, the surveillance behaviours of intra-lesional microglia and their avid response to the delivered NPs. Such microglial clearance of nanotherapeutic agents is an important area for CNS drug discovery and nanotechnology research, but the rate of progress in this field is hampered by the absence of available experimental models in which the immune cell component of pathology sites can be adequately and easily mimicked. We consider that our model can serve as a valuable tool offering the technical ease of in vitro handling, with the pathological complexity of injury sites. Consequently, we consider that demonstration of the handling of nanoparticles by slice microglia, with proof of similarities to the in vivo situation (as shown in this study) is an important outcome, and can be predicted to be of high use in translational and pharmaceutical studies. This could be via the use of immune suppressive treatment (that can alter microglial numbers/function, as shown here), the use of the so called "stealth coatings" to evade immune cell uptake or even stem cell transplantation that has been suggested to reduce immune activation, highlighting the versatility of our model for therapeutic testing.

Importantly, we prove using the model that administration of an anti-inflammatory drug (DEX) that is currently used widely in clinical neurology, including for the treatment of acute $\mathrm{SCl}$, can reduce the infiltration of the injury site by microglia, a phenomenon that is entirely consistent with the reported effects of DEX in vivo [30]. In turn this reduction in the cellular response of microglia was clearly shown to limit their sequestration of NPs. This highlights the utility of our "reductionist" model for the developmental testing of pharmaceutical strategies to limit the sequestration of therapeutic NPs by neural immune cells.

A few different approaches have been taken to mimic SCl pathology in vitro including: monoculture systems (primary and cell lines), co-culture systems, microfluidic devices and organotypic slice cultures [31-37]. A number of factors must be taken into consideration when evaluating the utility of each model system including the ability to mimic intercellular 
uptake dynamics, definition of cellular ratios to model specific CNS regions/disease states, standardisation of protein coronas around introduced nanoparticles, ease of nanoparticle delivery, and amenability to various (including real-time) imaging techniques [9]. Microfluidics models usually (and mono-cultures by definition) do not contain all the major cell types present in the CNS and thus fail to mimic the complex multi-faceted components of $\mathrm{SCl}$ pathology, which limits their value in the context of NP uptake experiments. Co-culture systems often do not contain microglial cells, a significant omission given their dominant uptake profiles, and usually also lack simulated injury [9, 14]. A few laboratories have simulated $\mathrm{SCl}$ in organotypic spinal cord slice cultures, which contain multiple cell types within in vivo-relevant cytoarchitecture. However, two of these models were established by weight drop onto slices, which is not suitable for assessing NP uptake in clearly-demarcated lesion sites, and while these models mimicked some of the responses to injury in vivo overall the characterisation of pathology was basic (e.g. cell death) [33,36]. Another SCI model has been established based on induction of complete transecting injury in parasagittal organotypic spinal cord slices from neonatal mice [23]. However, cardinal neuropathological responses were only characterised in the neurons, neglecting neuroglial responses. An elegant model has been established that contains neurons and the major neuroglial cells, by seeding dissociated rat embryonic spinal cord on top of an astrocyte monolayer [37]. The mechanical induction of injury resulted in a lesion site with many of the cardinal pathological responses displayed by multiple cell types (demyelination, reactive astrocytosis, neurite degeneration and, importantly, microglial infiltration). This model is better defined and the induced injury produces a lesion site better-suited to NP uptake studies than the other models reported. However, we suggest that the cytoarchitecture of the spinal cord and potentially, therefore, some of the complex cellECM signalling present in vivo, is better mimicked in organotypic slice cultures. Indeed, in the context of model development for laboratory research, it has recently been suggested that the best experimental predictability is offered by organotypic slice models that can replicate the microenvironment of the donor tissue [38]. Our model is consistent with this paradigm. We consider therefore that the system developed by ourselves can provide clinically relevant insights into the fate of NPs delivered to neuropathology sites. Our injury-simulating model offers major benefits for regenerative neurology as a neuromimetic screening system given: (a) The full complement of neural cell subclasses is present in the slice arrays and can be easily detected using standard procedures; (b) the use of humane procedures to derive tissue and reduce reliance on live animal experiments; (c) the simplicity/ cost effectiveness of the methods and reagents used;

(d) several slices (up to seven) can be derived from a single animal at the donor ages used, significantly reducing animal usage; (e) reduced biological variabi- lity as both experimental and control slices can be derived from the same animal; (f) the derivation of organotypic spinal cord slices from human tissue has been proved feasible highlighting the clinical utility of the model [39]; (g) the ease of experimental manipulation and compatibility of the model with a wide range of imaging methods, including time lapse and confocal microscopy. The compatibility of time- lapse imaging whilst retaining slice viability was verified by live/dead staining; the capture of cellular NP uptake events within slice lesion sites also proved feasible. Introduction of NPs into the injured tissue was simple and rapid to perform, demonstrating the versatility of the injury model, which can therefore be used to study cellular interactions with a wide range of NPs/materials. Further, post-culture immunostaining of slices facilitated the direct identification of timelapse imaged cells and permitted the assessment of NP uptake across different cell types. Less obviously, a single growth medium is required to culture the slices, overcoming issues related to differential biomo- lecular corona formation when different biological media are used to culture individual cell types [9]. This can significantly affect NP uptake and is a major confounding factor in comparative and cross-cellular studies of NP uptake $[9,10]$.

While microglia will present a critical challenge for delivery strategies to the CNS, this is currently a poorly studied area in neuro-nanotechnology. Such impediments to the clinical translation of therapeutic nanoparticles raise important issues regarding the 
necessity to develop interventions to overcome the microglial barrier (in order to enhance bioavailability to other target neural cell types in the CNS). Clearly, the use of immunosuppressive or immunomodulatory therapies to reduce microglial activity could constitute one such clinically relevant approach. In this regard, the corticosteroid DEX is commonly prescribed as an immunosuppressive therapy following injuries to the CNS $[40,41]$ and acts by inhibiting microglial proliferation [42], reducing their migratory capabilities [43] and potentially increasing microglial apoptosis $[44,45]$. In keeping with this role of DEX in vivo, we observed a significant reduction in the numbers of microglia in slice lesion sites. The extent of NP accu- mulation within microglia was also reducedwhich would have predicted increased extracellular availabi- lity of NPs to other neural cell types. However, in our study we observed only the suggestion of an increase in astrocyte labelling with no apparent enhancement of particle uptake in other neural cell types. The reasons for this lack of effect are not clear at present, and DEX-induced impairment of endocytic processes in other (nonmicroglial) neural cell types cannot be ruled out. The use of different immunosuppressive drugs and longer treatment regimens would prove interesting in this regard. In this context, we recently showed that even when a small population of microglia (less than $5 \%$ ) are present within high purity monocultures of astrocytes, oligodendrocyte precursors and oligodendrocytes, NP uptake by the non-microglial cells is dramatically limited due to avid microglial sequestration [10]. This underscores the highly responsive nature of the microglial response, for identification and removal of foreign materials from the CNS. Consequently, even a small residual population of microglia within the lesion sites persisting after DEX treatment may have been sufficient to dramatically limit particle uptake in other cell types.

Nonetheless, our findings do highlight the utility of our model as a screening system for strategies that promote evasion of the neural immune component, in order to optimise the use of NPbased approaches for neuropharmacology and nanotherapeutics. We consider that the use of similar experimental models based on 3-D organotypic slices derived from other types of biological tissues, has great potential for the screening of nanotherapeutic agents for a spectrum of disease and injury. As such, we consider that our approach has high relevance to the nanotechnology community in the developmental testing of pharmaceutical interventions based on nanotherapeutic agents.

\section{Conclusions}

We demonstrate the multiple advantages offered by a 3-D simulation of SCl (based on organotypic slice cultures of spinal cord tissue), to examine the fate of NPs delivered into areas of neural pathology, specifically their uptake by the resident immune cells. Dynamic live-cell imaging and histological assessment following NP delivery into slice injury sites revealed in real-time the well-documented phenomenon of competitive sequestration of particles by infiltrating microglia-a process that markedly reduced uptake in other neural cells in the injury sites. Use of immunosuppressive DEX-treatment proved to be an effective means to reduce microglial sequestration of particles. These results provide a real-time demonstration of the major intrinsic neural immune barrier that exists in the context of CNS-targeted nanotherapeutics. Our biological model therefore provides an alternative platform to test strategies to promote evasion of microglial clearance of biomaterials whilst reducing the use of live animal injury models for developmental testing of such interventions, in nanotechnology research.

\section{Acknowledgements}

The study is supported by the Engineering and Physical sciences research council (EPSRC; UK) Centre for Doctoral Training (CDT) in Regenerative Medicine (No. EP/F500491/1). S. I. J. was funded by an EPSRC Engineering Tissue Engineering and Regenerative Medicine (E-TERM) Landscape Fellowship (No. EP/I017801/1). The authors of this article report no involvement by the sponsors during the preparation of the manuscript or any conflict of interest.

Electronic Supplementary Material:
Supplementary material (three time lapse videos
S1-S3, showing


microglial motility and proliferation within lesion sites \pm NPs) is available in the online version of this article at http://dx.doi.org/10.1007/s12274-016-11257.

\section{References}

[1] Hoff, D.; Sheikh, L.; Bhattacharya, S.; Nayar, S.; Webster,

T. J. Comparison study of ferrofluid and powder iron oxide nanoparticle permeability across the blood-brain barrier. Int. J. Nanomedicine 2013, 8, 703-710.

[2] Bhaskar, S.; Tian, F. R.; Stoeger, T.; Kreyling, W.; de la Fuente, J. M.; Grazú, V; Borm, P.; Estrada, G.; Ntziachristos, V.; Razansky, D. Multifunctional nanocarriers for diagnostics, drug delivery and targeted treatment across blood-brain barrier: Perspectives on tracking and neuroimaging. Part. Fibre Toxicol. 2010, 7, 3.

[3] David, A. E.; Cole, A. J.; Yang, V. C. Magnetically targeted nanoparticles for brain tumor therapy: What does the future hold? Nanomedicine 2011, 6, 11331135.

[4] Wong, H. L.; Wu, X. Y.; Bendayan, R. Nanotechnological advances for the delivery of CNS therapeutics. Adv. Drug Deliv. Rev. 2012, 64, 686700.

[5] Nayak, D.; Roth, T. L.; McGavern, D. B. Microglia develop- ment and function. Annu. Rev. Immunol. 2014, 32, 367-402.

[6] Lee, G.; Dallas, S.; Hong, M.; Bendayan, R. Drug transporters in the central nervous system: Brain barriers and brain parenchyma considerations. Pharmacol. Rev. 2001, 53, 569-596.

[7] Tremblay, M.-̇̇.; Stevens, B.; Sierra, A.; Wake, H.; Bessis, A.; Nimmerjahn, A. The role of microglia in the healthy brain. J. Neurosci. 2011, 31, 16064-16069.

[8] Nimmerjahn, A.; Kirchhoff, F.; Helmchen, F. Resting microglial cells are highly dynamic surveillants of brain parenchyma in vivo. Science 2005, 308, 13141318.

[9] Jenkins, S. I.; Roach, P.; Chari, D. M. Development of a nanomaterial bio-screening platform for neurological applications. Nanomedicine 2015, 11, 77-87.

[10] Pinkernelle, J.; Calatayud, P.; Goya, G. F.; Fansa, H.; Keilhoff, G. Magnetic nanoparticles in primary neural cell cultures are mainly taken up by microglia. BMC Neurosci. 2012, 13, 32.

[11] Maysinger, D.; Behrendt, M.; Lalancette-Hébert, M.; Kriz, J. Real-time imaging of astrocyte response to quantum dots: In vivo screening model system for biocompatibility of nano- particles. Nano Lett. 2007, 7, 2513-2520.

[12] Fleige, G.; Nolte, C.; Synowitz, M.; Seeberger, F.; Kettenmann, $H_{\text {. }}$; Zimmer, C. Magnetic labeling of activated microglia in experimental gliomas. Neoplasia 2001, 3, 489-499.

[13] van Landeghem, F. K. H.; Maier-Hauff, K.; Jordan, A.; Hoffmann, K. T.; Gneveckow, U.; Scholz, R.; Thiesen, B.; 
Brück, W.; von Deimling, A. Post-mortem studies in glioblastoma patients treated with thermotherapy using magnetic nanoparticles. Biomaterials 2009, 30, 52-57.

[14] Jenkins, S. I.; Pickard, M. R.; Furness, D. N.; Yiu, H. H. P.; Chari, D. M. Differences in magnetic particle uptake by CNS neuroglial subclasses: Implications for neural tissue engineering. Nanomedicine 2013, 8, 951-968.

[15] McGonigle, P.; Ruggeri, B. Animal models of human disease: Challenges in enabling translation. Biochem. Pharmacol. 2014, 87, 162-171.

[16] Talac, R.; Friedman, J. A.; Moore, M. J.; Lu, L.; Jabbari, E.; Windebank, A. J.; Currier, B. L.; Yaszemski, M. J. Animal models of spinal cord injury for evaluation of tissue engineering treatment strategies. Biomaterials. 2004, 25, 1505-1510.

[17] Vieira de Castro, A. C.; Olsson, I. A. S. Does the goal justify the methods? Harm and benefit in neuroscience research using animals. Curr. Top. Behav. Neurosci. 2015, 19, 47-78.

[18] Sauer, U. G. Animal and non-animal experiments in nanotechnology-The results of a critical literature survey. ALTEX 2009, 26, 109-128.

[19] Zhang, H.; Jarjour, A. A.; Boyd, A.; Williams, A. Central nervous system remyelination in culture-A tool for multiple sclerosis research. Exp. Neurol. 2011, 230, 138-148.

[20] Weightman, A. P.; Pickard, M. R.; Yang, Y.; Chari, D. M. An in vitro spinal cord injury model to screen neuroregenerative materials. Biomaterials 2014, 35, 3756-3765.

[21] Jeffery, N. D.; McBain, S. C.; Dobson, J.; Chari, D. M. Uptake of systemically administered magnetic nanoparticles (MNPs) in areas of experimental spinal cord injury (SCI). J. Tissue Eng. Regen. Med. 2009, 3, 153-157.

[22] Stoppini, L.; Buchs, P. A.; Muller, D. A simple method for organotypic cultures of nervous tissue. J. Neurosci. Methods 1991, 37, 173-182.

[23] Bonnici, B.; Kapfhammer, J. P. Spontaneous regeneration of intrinsic spinal cord axons in a novel spinal cord slice culture model. Eur. J. Neurosci. 2008, 27, 2483-2492.

[24] Pickard, M. R.; Chari, D. M. Robust uptake of magnetic nanoparticles (MNPs) by central nervous system (CNS) microglia: Implications for particle uptake in mixed neural cell populations. Int. J. Mol. Sci. 2010, 11, 967-981.

[25] Pickard, M. R.; Jenkins, S. I.; Koller, C. J.; Furness, D. N.; Chari, D. M. Magnetic nanoparticle labeling of astrocytes derived for neural transplantation. Tissue Eng. Part C Methods 2011, 17, 89-99.

[26] Cooper, J. A. Background Subtraction to Obtain
Total Fluorescence per Cell, using ImageJ and Excel [Online], 2008; pp 1-8. http://www.cooperlab.wustl.edu/ LabMethodsReagentsOperations/Background Subtract Total Fluor per Cell/Instructions.pdf (accessed Aug 20, 2015). 
[27] Nehmé, A.; Lobenhofer, E. K.; Stamer, W. D.; Edelman,

J. L. Glucocorticoids with different chemical structures but similar glucocorticoid receptor potency regulate subsets of common and unique genes in human trabecular meshwork cells. BMC Med. Genomics 2009, 2, 58.

[28] Sur, P.; Sribnick, E. A.; Patel, S. J.; Ray, S. K.; Banik, N. L. Dexamethasone decreases temozolomideinduced apoptosis in human gliobastoma T98G cells. Glia 2005, 50, 160-167.

[29] McCarthy, K. D.; de Vellis, J. Preparation of separate astroglial and oligodendroglial cell cultures from rat cerebral tissue. J. Cell Biol. 1980, 85, 890-902.

[30] Nguyen, K. B.; McCombe, P. A.; Pender, M. P. Increased apoptosis of $\mathrm{T}$ lymphocytes and macrophages in the central and peripheral nervous systems of Lewis rats with experimental autoimmune encephalomyelitis treated with dexamethasone. J. Neuropath. Exp. Neur. 1997, 56, 58-69.

[31] East, E.; Golding, J. P.; Phillips, J. B. Engineering an integrated cellular interface in three-dimensional hydrogel cultures permits monitoring of reciprocal astrocyte and neuronal responses. Tissue Eng. Part C Methods 2012, 18, 526-536.

[32] Kim, H. J.; Park, J. W.; Park, J. W.; Byun, J. H.; Vahidi, B.; Rhee, S. W.; Jeon N. L. Integrated microfluidics platforms for investigating injury and regeneration of CNS axons. Ann. Biomed. Eng. 2012, 40, 1268-1276.

[33] Krassioukov, A. V.; Ackery, A.; Schwartz, G.; Adamchik, Y.; Liu, Y.; Fehlings, M. G. An in vitro model of neurotrauma in organotypic spinal cord cultures from adult mice. Brain Res. Protoc. 2002, 10, 60-68.

[34] Morrison, B., III; Saatman, K. E.; Meaney, D. F.; Mclntosh,

T. K. In vitro central nervous system models of mechanically induced trauma: A review. J. Neurotrauma 1998, 15, 911-928.

[35] Ravikumar, M.; Jain, S.; Miller, R. H.; Capadona, J. R.; Selkirk, S. M. An organotypic spinal cord slice culture model to quantify neurodegeneration. $J$. Neurosci. Methods 2012, 211, 280-288.

[36] Balentine, J. D.; Greene, W. B.; Bornstein, M. In vitro spinal cord trauma. Lab. Invest. 1988, 58, 93-99.
[37] Boomkamp, S. D.; Riehle, M. O.; Wood, J.; Olson, M. F.; Barnett, S. C. The development of a rat in vitro model of spinal cord injury demonstrating the additive effects of rho and ROCK inhibitors on neurite outgrowth and myelination. Glia 2012, 60, 441-456.

[38] Heinonen, T. Better science with human cell-based organ and tissue models. Altern. Lab. Anim. 2015, 43, 29-38.

[39] Jeong, D. K.; Taghavi, C. E.; Song, K. J.; Lee, K. B.; Kang,

H. W. Organotypic human spinal cord slice culture as an alternative to direct transplantation of human bone marrow precursor cells for treating spinal cord injury. World Neurosurg. 2011, 75, 533-539.

[40] Reynolds, R. M.; Seckl, J. R. Antenatal glucocorticoid treatment: Are we doing harm to term babies? J. Clin. Endocrinol. Metab. 2012, 97, 3457-3459.

[41] Burton, J. M.; O'Connor, P. W.; Hohol, M.; Beyene, J. Oral versus intravenous steroids for treatment of relapses in multiple sclerosis. Cochrane Database Syst. Rev. 2012, 12, CD006921.

[42] Ganter, S.; Northoff, H.; Männel, D.; Gebicke-Härter, P. J. Growth control of cultured microglia. J. Neurosci. Res. 1992, 33, 218-230.

[43] Zhou, Y.; Ling, E.-A.; Dheen, S. T. Dexamethasone suppresses monocyte chemoattractant protein-1 production via mitogen activated protein kinase phosphatase- 1 dependent inhibition of Jun N-terminal kinase and p38 mitogen-activated protein kinase in activated rat microglia. J. Neurochem. 2007, 102, 667-678.

[44] Jenkins, S. I.; Pickard, M. R.; Khong, M.; Smith, H. L.; Mann, C. L. A.; Emes, R. D.; Chari, D. M. Identifying the cellular targets of drug action in the central nervous system following corticosteroid therapy. ACS Chem. Neurosci. 2014, 5, 51-63.

[45] Li, M. Q.; Wang, Y. Y.; Guo, R. W.; Bai, Y.; Yu, Z. P. Glucocorticoids impair microglia ability to induce $T$ cell proliferation and Th1 polarization. Immunol. Lett. 2007, 109, 129-137. 


\section{Table of contents}

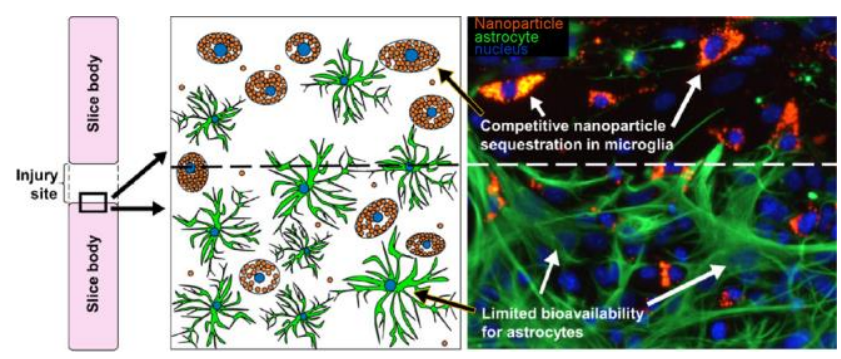

A "dish" model of spinal cord injury has been used to demonstrate the clearance of nanoparticles by neural immune cells in real time. This model shows high utility for evaluating novel nanoparticles and their handling by nervous system cells. 

